\title{
ResearchOnline@JCU
}

This is the Accepted Version of a paper published in the journal Developmental Psychobiology:

Rymer, Tasmin L., and Pillay, Neville (2013) Maternal care in the African striped mouse Rhabdomys pumilio: a behaviorally flexible phenotype that is modified by

experience. Developmental Psychobiology, 55 (3). pp. 265274.

http://dx.doi.org/10.1002/dev.21020 


\section{Maternal care in the African striped mouse Rhabdomys pumilio: a behaviourally flexible phenotype that is modified by experience}

Expression of maternal care. Rymer and Pillay

TASMIN L. RYMER ${ }^{1}$, NEVILLE PILLAY ${ }^{1 *}$

${ }^{1}$ School of Animal, Plant and Environmental Sciences, University of the Witwatersrand, Private Bag 3, Wits 2050, South Africa

T. Rymer: tasminrymer@gmail.com

N. Pillay: Neville.Pillay@wits.ac.za

* Corresponding author: N. Pillay

Address: School of Animal, Plant and Environmental Sciences, University of the Witwatersrand, Johannesburg, Private Bag 3, Wits 2050, South Africa

Tel: +27117176459

Fax: +27117176494

* This is the Accepted Version of a paper published in the journal Developmental Psychobiology:

Rymer, T. L. \& Pillay, N. 2013. Maternal care in the African striped mice Rhabdomys pumilio: a behaviorally flexible phenotype that is modified by experience. Developmental Psychobiology, 55, 265-274.

Note: First author is now at James Cook University: email Tasmin.Rymer@jcu.edu.au. 
The development of maternal care in mammals can be influenced by the type and quality of maternal care received. Using biparental striped mice Rhabdomys pumilio, we investigated whether development of maternal care is influenced by the mother during early rearing and by an adult female's experience and that of her mate. Offspring were raised in one of three treatments, by: both parents; mothers alone; and mothers separated from the father with a barrier. Since female striped mice increase their care when raising litters alone, which influences expression of parental care of their adult sons, we expected daughters to respond like sons. However, there was no treatment effect in the development of maternal care in daughters. In subsequent experiments, experienced and inexperienced females decreased care when raising their offspring with experienced but not inexperienced males. Therefore, maternal care in striped mice is modulated in response to prevailing environmental and social conditions.

Keywords: activational effects; early postnatal environment; maternal care; organizational effects; paternal care; Rhabdomys pumilio

\footnotetext{
* This is the Accepted Version of a paper published in the journal Developmental Psychobiology: Rymer, T. L. \& Pillay, N. 2013. Maternal care in the African striped mice Rhabdomys pumilio: a behaviorally flexible phenotype that is modified by experience. Developmental Psychobiology, 55, 265-274. 
The behavioral expression of maternal care in mammals can be influenced by maternal effects when young are in utero or during the early postnatal period (organizational effects: Liu et al., 1997; Meaney, 2001). Furthermore, females may change their maternal care behavior at a later stage, depending on prevailing environmental and social conditions (activational effects: Numan \& Insel, 2003).

Organizational effects create permanent, nonreversible, changes in neural substrates underlying behavior during early critical periods (i.e., pre- or perinatal; Elekonich \& Robinson, 2000; Phoenix, Goy, Gerall, \& Young, 1959; Young, Goy, \& Phoenix, 1964), while activational effects are usually reversible, and modify neural activity in pathways previously laid down by organizational effects, to alter the behavioral state in adulthood (Elekonich \& Robinson, 2000). Although activational hormonal effects stimulate the onset of maternal care in adult females (reviewed in Numan \& Insel, 2003), the maternal care phenotype may also be organized by mothers both directly, through hormonal transmission during gestation, or indirectly, through provision of maternal care postweaning (Soares et al., 2010). Direct alteration of offspring behavior occurs during embryonic development, where, for example, maternal hormones (reproductive hormones: e.g., estrogen: Goebelsmann, Roberts, \& Jaffe, 1972; stress hormones: e.g., cortisol: Van den Bergh, Mulder, Mennes, \& Glover, 2005) may be transferred across the placenta, thereby influencing the offspring's hormonal profile (and hence behavioral phenotype). The organizational effect of these hormones affects the individual's phenotype (Soares et al., 2010) by creating permanent changes in the neural substrates that underlie behavior (Elekonich \& Robinson, 2000). These organizational effects may even cause the development of behavior to proceed along different developmental trajectories between individuals within the same litter (Elekonich \& Robinson, 2000). 
Indirect alteration of offspring behavior may also occur during early postnatal development. Many studies have investigated the influence of the mother's care on the development and behavioral expression of maternal behavior in their daughters (Champagne, Diorio, Sharma \& Meaney, 2001; Curley, Champagne, Bateson, \& Keverne, 2008; Francis, Diorio, Liu, \& Meaney, 1999; Jia, Tai, An, \& Zhang, 2011). For example, Francis et al. (1999) and Champagne et al. (2001) showed that female rats that receive high levels of maternal care during the early postnatal period display greater maternal care provisioning themselves as adults, while female mandarin voles Microtus mandarinus show lower levels of maternal care if they received lower levels of parental investment (Jia et al., 2011).

Most studies have investigated development of maternal care in experimental settings where mothers raise young in the absence of a mate (e.g., Curley et al., 2008; Kikusui, Isaka, \& Mori, 2005). However, changes in social conditions (i.e., the presence of a mate or family group) can cause a shift in the behavioral phenotype of an individual, through activational effects altering an individual's behavioral state (Elekonich \& Robinson, 2000). Fewer studies have investigated how maternal care is behaviorally expressed when both parents contribute to offspring care.

Fathers of many biparental rodents play a crucial role in offspring development (e.g., California mice Peromyscus californicus, Gubernick \& Teferi, 2000; trumpet-tailed rats Octodon degus, Outscharoff, Helmeke, \& Braun, 2006) and survival (Djungarian hamsters Phodopus campbelli, Wynne-Edwards, 1995). For example, young California mice that are raised by their mothers only receive lower levels of licking and grooming, which subsequently influences cognitive development (Bredy, Lee, Meaney, \& Brown, 2004). Fathers can also influence the development of parental care in their offspring. Prairie vole Microtus ochrogaster mothers show greater levels of huddling and retrieving if they received high levels of these behaviors from their fathers during the early postnatal period (Stone \& 
Bales, 2010). Furthermore, males can influence the parental care behavior of their mates, which then influences behavioral development in their offspring. For example, female redbacked voles Clethrionomys gapperi decrease maternal care in the presence of a mate, as males reduce the maternal workload by providing care, and offspring raised in the presence of their fathers show a greater motivation to explore (venture out the nest at an earlier age) than young raised by mothers only (McGuire, 1997).

The striped mouse Rhabdomys pumilio is a small ( $\pm 40 \mathrm{~g}$ ) diurnal murid rodent with a widespread, although discontinuous, distribution across southern Africa (Skinner \& Chimimba, 2005). Males from the semi-arid Succulent Karoo region of South Africa are naturally paternal, displaying all parental behaviors shown by females (including huddling, licking, grooming, and retrieving pups), apart from nursing (Schradin \& Pillay, 2003). However, striped mice are facultatively biparental and females will nest and raise their young alone when vacant nest sites become available during low population density (Schradin, Köenig, \& Pillay, 2010). In captivity, mothers from this population almost fully compensate for a lack of care provided by their mates when they raise young alone (i.e., increase care by $1 \frac{1}{2}$ times, to levels similar to total care provided by both parents), which subsequently influences the development of paternal care in their sons: sons in litters raised by mothers alone show higher levels of paternal care as adults than sons raised by both parents (Rymer $\&$ Pillay, 2011).

Since maternal care in female rodents is known to be influenced by the level of maternal care provided by their mothers, either positively (Francis et al., 1999) or negatively (Curley et al., 2008; Jia et al., 2011), the aim of this study was to investigate whether compensation of maternal care by striped mouse mothers in the absence of fathers influences the development of parental care in their daughters, as reported in sons (Rymer \& Pillay, 2011). We predicted that the absence of the father and concomitant compensation by the mother would result in 
the expression of higher levels of maternal care in their adult daughters. If so, maternal care behavior would be organized (indirectly) by the behavior of the mother during early development. We had no a priori expectation that male striped mice would influence the maternal care behavior of their daughters (as they did not influence paternal care behavior of sons; Rymer \& Pillay, 2011).

\section{Methods}

\section{Experiment 1: Development of maternal care}

The primary aim of this study was to investigate whether mothers influence the development and later expression of maternal care in adult daughters, as they do in their sons. The experimental design, following Rymer \& Pillay (2011) and described below, also allowed us to investigate whether fathers influence the development and behavioral expression of maternal care in their daughters, as reported in prairie voles (Stone \& Bales, 2010). Male striped mice positively influence offspring growth (by huddling pups; Schradin \& Pillay, 2005) but we do not know whether the care they provide influences the development of maternal care in their daughters.

Subjects. Individuals used in this study were F1-F4 generation striped mice derived from Goegap Nature Reserve (Succulent Karoo, Northern Cape Province, South Africa; S 29 41.56, E 18 1.60), housed under partially controlled environmental conditions (14 L:10 D cycle, lights on at 0500 hours; $20-24^{\circ} \mathrm{C} ; 30-60 \%$ relative humidity).

Breeding pairs (all treatments; age at pairing (mean $\pm \mathrm{SE}$ ): males $8 \pm 0.49$ months; females $7 \pm 0.46$ months; weight at pairing (mean $\pm \mathrm{SE}$ ): males $78.24 \pm 2.22 \mathrm{~g}$; females 70.65 
$\pm 2.19 \mathrm{~g}$ ) were established and housed in glass tanks (46 cm x $30 \mathrm{~cm} \times 32 \mathrm{~cm})$. Tank floors were covered with a layer of wood shavings for bedding and a plastic nest box $(13 \mathrm{~cm} \times 9 \mathrm{~cm}$ $\mathrm{x} 10 \mathrm{~cm}$ ) was provided. A handful of dry grass, and approximately $5 \mathrm{~g}$ of paper towel, was provided twice weekly for nesting material. One cardboard roll/paper cup was provided weekly per mouse for behavioral enrichment. Subjects had access to water ad libitum and were fed approximately $5 \mathrm{~g}$ of mixed seed (sprinkled throughout the cage to stimulate foraging behavior) and $10 \mathrm{~g}$ of fresh fruit/vegetables daily per mouse.

Experimental Design. We used the same animals and experimental protocol as described by Rymer \& Pillay (2011). Briefly, the experiment was divided into two phases. The intention was to obtain three consecutive litters per breeding pair $(n=15)$ and randomly assign each litter to one of three different treatments. Nine pairs produced the required three litters, three pairs each produced two litters and three pairs each produced one litter only. Data from an additional seven pairs (two pairs produced two litters each and five pairs produced one litter each) were distributed among all treatments to achieve the required sample size of 15 per treatment. In Phase 1, breeding pairs were subjected to three treatments in random sequence: (1) mother + father $(M+F)-$ both parents raised their young together until weaning (21 days of age); (2) mother + father separated (M/F) - the father was separated from the mother by inserting a wire mesh barrier (30 $\mathrm{cm} \times 32 \mathrm{~cm}, 1 \mathrm{~cm} \times 1 \mathrm{~cm}$ squares) into the tank and placing the male and female on opposite sides. Mothers raised young alone but in visual, olfactory and auditory contact with fathers; and (3) mother alone (M - F) - the father was removed from the mother and housed in a separate cage in a separate room. In the $\mathrm{M}-\mathrm{F}$ and $\mathrm{M} / \mathrm{F}$ treatments, males and females were separated a few days prior to parturition. All mothers had prior breeding experience, which we accounted for statistically in the data analyses. At weaning, offspring were housed in same-sex sibling pairs in holding cages (42 cm x $26 \mathrm{~cm} \mathrm{x}$ 
$14 \mathrm{~cm}$ ) until sexual maturity (approximately 90 days of age). In Phase 2, one adult daughter $($ daughter $=\mathrm{D})$ from each litter per treatment was randomly selected and paired with an unrelated mate of approximately the same age, resulting in three treatments: $\mathrm{DM}+\mathrm{F}, \mathrm{DM} / \mathrm{F}$, DM - F (daughter from $M+F, M / F$ and $M-F$, respectively). All daughters were sexually naïve prior to pairing. Pairs were housed as described above and all Phase 2 females raised their own first litter together with their mates. We video taped all pairs (Phase 1 and Phase 2) every second day (for a total recording of 6 days) and scored parental care of mothers and daughters (all treatments), and fathers $(\mathrm{M}+\mathrm{F}, \mathrm{DM}+\mathrm{F}, \mathrm{DM} / \mathrm{F}, \mathrm{DM}-\mathrm{F})$. Parental care (for all Phase 1 and Phase 2 pairs) was scored following Schradin \& Pillay (2003): time spent in close proximity $(<2 \mathrm{~cm}$ ) of pups (designated “near"), which facilitates the mother's interaction with pups, thereby promoting maternal care (as seen in prairie voles, Olazábal \& Young, 2005); huddling; grooming pups (includes sniffing and licking); and retrieving pups. As we could not differentiate between nursing and huddling pups for maternal care, we grouped the data and collectively classified it as huddling (as described by Schubert, Pillay, \& Schradin, 2009). The results for retrieval behavior are not included here as the incidence was rare and did not vary among the treatments $(p>0.05)$.

We recorded the masses (nearest $0.1 \mathrm{~g}$ ) of all male and female pups in litters, produced in both phases, every day after birth for the first 7 days, and every 3 days thereafter until day 21 . These data were used to calculate growth rates, using the formula: (LN mass day 21 - LN mass day 1)/20 days.

Statistical Analysis. We used Statistica 7.1 (Statsoft, Inc., www.statsoft.com) for all analyses. All parental care data met the assumptions of normality (Shapiro-Wilk's test) and homogeneity of variances (Levene's test). We first analyzed the data set for the three behaviors (near, huddling, grooming) for maternal (mothers and daughters) and paternal 
(fathers) care over the 6 days of video-recording (designated "time"), as well as offspring growth rates, with the variance components analysis, using the restricted maximum likelihood (REML) method to assess the effects of random factors (litter order, breeding pair identity) on the behavioral variables. Both random factors were not significant predictors of parental care or growth rate $(p>0.05)$ for all cases, indicating that neither parental care nor growth rate changed with maternal parity. Therefore, they were excluded in further analyses.

We then used a General Linear Model (GLM) with repeated measures, multivariate design, to analyze the data. Treatment was the categorical predictor, the three behaviors were the dependent variables, time was the repeated measures variable and litter size was the covariate. Specific differences in the main effects were identified using Fisher's HSD post hoc tests. We used orthogonal polynomial decomposition for linear and quadratic components to assess whether or not changes in behavior over time were random for treatment x time interactions (see Rymer \& Pillay, 2011). Parental care displayed by mothers (Phase 1) and daughters (Phase 2) was also compared using a linear regression.

We arcsine transformed the growth rate data of male and female offspring and analyzed growth rates for each sex separately using a GLM with repeated measures design, in which treatment was the categorical predictor, growth rate (male or female) was the repeated measures variable and litter size and sex ratio (M:F) were the covariates. All tests were twotailed and the model-level significance was determined at $\alpha=0.05$.

Results. Treatment, time, and the interaction between treatment and time were all significant predictors of maternal care (Table 1). Mothers raising young together with their mates $(\mathrm{M}+$ F) spent the most time near pups, followed by daughters (DM - F, DM + F, DM/F), while mothers raising young alone (M - F; M/F) spent the least time near pups (Table 1; Fig. 1). In contrast, compared to all other treatments, $\mathrm{M}-\mathrm{F}$ and $\mathrm{M} / \mathrm{F}$ mothers spent the most time 
huddling pups, followed by daughters (DM - F, DM + F, DM/F), while $\mathrm{M}+\mathrm{F}$ mothers spent the least time huddling pups (Table 1; Fig. 1). There was a graded response in grooming with $\mathrm{M}-\mathrm{F}$ and $\mathrm{M} / \mathrm{F}$ mothers showing the highest levels of grooming and $\mathrm{M}+\mathrm{F}$ mothers showing the lowest levels of grooming (Table 1; Fig. 1). Daughters (DM - F, DM/F, and DM + F) occupied an intermediate position.

For both Phase 1 and Phase 2 mothers, huddling behavior was greatest during the early postnatal period (Days 1 and 3) and lowest during the late postnatal period (Days 9 and 11: Table 1; Fig. 1). In contrast, time spent near pups was lowest during the early postnatal period and greatest in the late post-natal period, indicating an inverse relationship with huddling behavior (Table 1; Fig. 1). Phase 1 mothers showed higher levels of grooming during the midpostnatal period (Days 3-9), while Phase 2 mothers showed higher levels of grooming during the late postnatal period (Days 9 and 11: Table 1; Fig. 1).

For the treatment $\mathrm{x}$ time interaction, polynomial components were not significant for the time spent near (quadratic: $t=1.34, p=0.182$ ), huddling (quadratic: $t=0.18, p=0.240$ ), and grooming (linear: $t=0.54, p=0.593$; quadratic: $t=1.71, p=0.091$ ) pups. However, there was a significant linear component for the time spent near $(t=2.50, p=0.014)$ and huddling $(t=3.88, p<0.000)$ pups. Overall, the general pattern in the relationship between treatment and time is random. Maternal care was not influenced by litter size (Table 1).

There was no significant relationship between the maternal care provided by mothers (Phase 1) and their daughters (Phase 2) for being near (linear regression: $R^{2}=0.36 ; F_{1,126}=$ 2.71, $p=0.102)$, huddling $\left(R^{2}=0.26 ; F_{1,126}=1.21, p=0.273\right)$, or grooming $\left(R^{2}=0.16 ; F_{1,126}\right.$ $=0.767, p=0.383)$ pups.

The growth rates of male and female offspring in each of the two phases are presented in Table 2. Growth rates were not significantly different in either phase, suggesting that neither the presence/absence of the father (Phase 1), nor the rearing history of daughters (Phase 2), 
influenced offspring growth. Furthermore, this suggests that there is no differential investment by mothers in either sons or daughters (Table 2). Litter size and sex ratio were also not significant predictors of growth (Table 2).

\section{Experiment 2: Effect of female experience on the behavioural expression of maternal care}

The results obtained for the first experiment were unexpected because daughters did not respond to maternal compensation by increasing parental care, as their brothers did. We therefore conducted two further experiments a posteriori to investigate possible explanations for the results. Daughters had a different behavioral pattern to their mothers, showing consistently high levels of care, regardless of treatment (Fig. 1). All mothers in Phase 1 were maternally experienced and decreased their level of huddling and grooming when raising young with a mate, indicating that: (1) males helped to reduce the maternal workload; and/or (2) females alter care as they age and gain parenting experience. Since daughters (Phase 2) also raised their young with a mate, but did not reduce care, it would appear that maternal care might change as females become experienced mothers. A study on reindeer Rangifer tarandus (Weladji et al., 2006) has also suggested a change in parental care with experience. Therefore, we designed a second experiment to establish whether the level of maternal care shown by female striped mice was fixed (i.e., they would show the same amount of care to a second litter as to the first litter) or changed with maternal parity (i.e., as they became more experienced mothers).

Subjects and Experimental Design. We used 20 female striped mice (not used in Experiment 1) reared under the same breeding conditions as described above for Phase 2 females, that is, 
10 females originated from pairs raising young together $(\mathrm{M}+\mathrm{F})$ and 10 females originated from mothers raising young alone $(\mathrm{M}-\mathrm{F})$, resulting in two conditions: $\mathrm{DM}+\mathrm{F}$ and $\mathrm{DM}-\mathrm{F}$. We excluded daughters from the $\mathrm{M} / \mathrm{F}$ condition as values for maternal care were intermediate to those from $\mathrm{M}+\mathrm{F}$ and $\mathrm{M}-\mathrm{F}$ and the conditions of $\mathrm{M} / \mathrm{F}$ did not test for differences in maternal care per se (i.e., the treatment was designed to assess the effect of the close proximity of the father). At weaning, daughters (DM + F and DM - F) were housed in holding cages in same sex sibling pairs. At maturity, daughters were paired with unrelated same age reproductively naïve males and raised their first litter as described for Phase 2 females (Experiment 1). Behavioral observations were identical to those described in Experiment 1. As we were mostly interested in establishing whether there was a pattern between first and second litter investment by mothers, we did not distinguish between maternal care behaviors statistically (as for Experiment 1), but rather summed the durations of all care-giving behaviors (near, huddling, grooming) into one category of maternal care. Females remained with their mates and raised a second litter after weaning of the first litter (inter litter interval approx. 23 days) and behavioral observations for mothers were repeated as described previously.

Statistical Analysis. We analyzed the data set for maternal care for each litter over the 6 days of video recording and between the first and second litters (designated "litter number") with a repeated measures GLM. Treatment was the categorical predictor, maternal care was the dependent variable and litter number was the repeated measures variable. Litter sizes (for both litters) were covariates. Specific differences were identified using Fisher's HSD post hoc tests. All tests were two-tailed and the model-level significance was determined at $\alpha=0.05$. 
Results. There was a significant effect of litter number (i.e. first or second litter) on maternal care $\left(F_{6,11}=4.31, p=0.018\right.$; Fig. 2$)$. Post hoc tests showed that, regardless of treatment, mothers decreased the amount of care provided when they reared their second litter, except on the ninth day postpartum (Fig. 2). Treatment $\left(F_{6,11}=1.44, p=0.282\right)$, litter size (first litter 1: $F_{6,11}=0.50, p=0.793$; second litter: $\left.F_{6,11}=0.64, p=0.700\right)$ and the interaction between litter number and treatment $\left(F_{6,11}=1.71, p=0.209\right)$ were not significant predictors of maternal care.

\section{Experiment 3: Effect of mate experience on the behavioural expression of maternal care}

Daughters decreased maternal care as they gained parenting experience, but this does not provide an adequate explanation for why they initially (Experiment 1 and first litter in Experiment 2) showed a different behavioral response to their brothers (Rymer \& Pillay, 2011), as all daughters, like sons, were maternally naïve when rearing their first litter. Since females increase their levels of maternal care when their mates are absent or housed separately, but in close proximity (i.e., compensate when mates are absent; Rymer \& Pillay, 2011), we hypothesized that the level of maternal care in striped mice may also be affected by the motivation of their mates to provide paternal care. Therefore, it is possible that females reduce parental care when males show a high motivation to provide care (i.e., they have cared for a litter before), and males may reduce the maternal workload by providing care (e.g., redbacked voles, McGuire, 1997). Therefore, we designed a third experiment to establish whether the level of maternal care shown by naïve female striped mice changes in response to male motivation to provide care, and predicted that females would decrease their care when raising young with paternally experienced males. 


\section{Subjects and Experimental Design.}

We used 20 inexperienced female striped mice (not used in Experiment 1 or 2) reared under the same breeding conditions as described above for Phase 2 females. All females originated from pairs raising young together $(\mathrm{M}+\mathrm{F})$, resulting in a $\mathrm{DM}+\mathrm{F}$ condition. We excluded daughters from the M - F condition as we did not want to introduce the potential confounding effects of social rearing condition on maternal care. At weaning, daughters were housed as described in Experiment 2. At sexual maturity, 10 inexperienced daughters were paired with unrelated, same age inexperienced males and raised their first litter as described for Phase 2 females in Experiment 1 (designated "inexperienced"). Females remained with their mates and raised a second litter (after weaning of the first litter), to provide a comparison with results obtained from Experiment 2 (designated "experienced"). The other 10 inexperienced daughters were paired with an unrelated older experienced male (>120 days old) and raised one litter as described for Phase 2 females in Experiment 1 (designated "older male"). These males had raised at least 2 litters in captivity prior to the experiment. Behavioral observations were identical to those described in Experiment 1. As for Experiment 2, we were mostly interested in establishing whether there was a relationship between male experience and maternal care, and therefore we did not distinguish between the components of maternal care behaviors.

\section{Statistical Analysis.}

We first analyzed the data set for maternal care of the three treatment groups (inexperienced, experienced, older male) over the 6 days of video recording with the variance components analysis, using the REML method to assess the effects of individual identity (i.e., female identity; random factor) on behavior. Individual identity was not a significant predictor of parental care $(p>0.05)$ for all cases. Therefore, individual identity was excluded in further 
analyses. We then analyzed the data set for maternal care for each treatment group over time with a repeated measures GLM. Treatment was the categorical predictor, maternal care was the dependent variable, time was the repeated measures variable and litter size was the covariate. Specific differences were identified using Fisher's HSD post hoc tests. All tests were two-tailed and the model-level significance was determined at $\alpha=0.05$.

Results. Treatment was a significant predictor of maternal care (Table 3; Fig. 3). Post hoc tests showed that inexperienced mothers raising their first litter with an older experienced male showed a similar lower level of maternal care to experienced mothers raising young with experienced males (Fig. 3). In contrast, inexperienced mothers raising their first litter with inexperienced males showed the highest levels of maternal care (Fig. 3). Litter size and the interaction between treatment and time were not significant predictors of maternal care (Table 3).

\section{Ethical Note}

All animals received environmental enrichment (as described above) and the experimental procedures had no obvious negative effects on striped mouse welfare. Individuals were returned to the striped mouse colony after experiments for use in other studies. This study complied with the current laws and regulations in South Africa and was approved by the Animal Ethics Screening Committee of the University of the Witwatersrand (clearance no. 2005/51/2A). 


\section{Discussion}

Contrary to expectations, daughters showed a consistently high level of care, regardless of rearing environment, indicating firstly that maternal compensation does not influence the development of maternal care and secondly an absence of organizational effects. Our results contrast with those of other studies in mandarin voles, prairie voles, laboratory mice, and rats, showing that variations in maternal care influence the development of maternal care in their daughters (Curley et al., 2008; Francis et al., 1999; Jia et al., 2011; Kikusui et al., 2005; Stone \& Bales, 2010). In Experiment 1, although Phase 1 mothers showed a reduction in their level of care (huddling and grooming) when their mates were present $(\mathrm{M}+\mathrm{F})$, daughters maintained an exaggerated level of care that would be expected if they responded like mothers raising young in the absence of a mate $(\mathrm{M}-\mathrm{F})$.

Organizational effects have traditionally been viewed as acting during early critical periods to produce an irreversible, or fixed, behavioral phenotype (Elekonich \& Robinson, 2000). Maternal care behavior has been shown to be organized by mothers both directly, through hormonal transmission during gestation (Goebelsmann et al., 1972; Van den Bergh et al., 2005) and indirectly, through provision of maternal care post-weaning (Soares et al., 2010). While striped mice sons raised only by their mothers increased their level of paternal care as adults as a likely consequence of maternal compensation (Rymer \& Pillay, 2011), daughters were unaffected. Therefore, differences in the development and expression of parental care between male and female striped mice might reflect sex differences in the timing of organization of parental care behavior. Sexual differences in behavior due to putative organizational effects have been used to explain behavioral development in other rodents (see Galea, Kavaliers, \& Ossenkopp, 1996). While we cannot rule out that brothers and sisters might vary in their response to levels of care provided by their mothers, for 
example, through levels of grooming received (Rymer \& Pillay, 2011), it appears that, in sons, parental care behavior is indirectly organized by stimuli from the mother during early postnatal development. In contrast, maternal care behavior in daughters is not influenced by maternal care during early postnatal development but may be organized by other means, (e.g., in utero) since daughters showed consistently high levels of care, regardless of rearing conditions. However, if maternal care behavior in female striped mice were organized in utero, we would then expect all females, including Phase 1 mothers, to show consistently high levels of maternal care. This was not the case, however, indicating that an organizational effect cannot exclusively explain the behavioral expression of maternal care in female striped mice.

Lactogenic (e.g., prolactin) and steroid hormones (e.g., estrogen and progesterone) are required to stimulate (i.e., activate) the onset of maternal care (reviewed in Numan \& Insel, 2003). These hormones most likely mediate the maternal response in females. However, changes in hormone secretion may be driven by changing environmental and social conditions, which can subsequently influence reproductive behavior. For example, Syrian hamsters Mesocricetus auratus maintained under short ( $<12.5$ hours) light periods show a decrease in the concentration of circulating gonadotropins and sex steroids, resulting in the cessation of reproductive behavior (reviewed in Prendergast, Zucker, \& Nelson, 2009). In addition, estrogens act as neuromodulators (which regulate ongoing neural activity to promote context-specific behaviors; Libersat \& Pflüger, 2004), adjusting behavioral expression in response to variations in social context, such as the presence of males in the breeding season (Oliveira, 2009). Furthermore, changes in experience can also alter the expression of estrogen receptor a (ER $\alpha$ : Song et al., 2010) and oxytocin receptor levels (Champagne et al., 2001), changing parental care behavior. Our studies indicate that as striped mice become experienced mothers, their parental care behavior is altered, suggesting 
a change in either hormonal influence or changes in the estrogen-inducible central oxytocin receptors. Reindeer become better mothers as they become more experienced (Weladji et al., 2006) and female striped mice alter their mate preference behavior when they become sexually experienced (Rymer \& Pillay, 2010). In our study, all Phase 2 daughters were naïve and inexperienced. As they became more experienced (raising a second litter, Experiment 2), they decreased their level of care. This suggests that maternal care is differentially activated in multiparous female striped mice. However, the mates of Phase 2 daughters were also becoming more experienced, indicating that maternal experience alone cannot fully explain a decrease in care by mothers from their first to their second litters and that there may be a complementary role by the mate.

Male striped mice show high levels of paternal care in nature (Schradin \& Pillay, 2004) and captivity (Rymer \& Pillay, 2011) in equal measure (huddling, licking, and time spent in the nest: Schradin \& Pillay, 2003; Schubert et al., 2009) to maternal care. While organizational effects may influence the high levels of maternal care initially, and females can alter the level of care provided through experience, it is also likely that male caring experience modulates the behavioral expression of maternal care in females. McGuire (1997) found that female red-backed voles reduce their level of maternal care in the presence of experienced mates, as males help to reduce maternal workload. Similarly, we found that inexperienced female striped mice raising young with experienced males show lower levels of maternal care than inexperienced females raising their young with inexperienced males (Experiment 3). This suggests that male experience can also alter the behavioral expression of maternal care in female striped mice.

The Succulent Karoo is an unpredictable and highly variable environment, with low annual rainfall and high temperature fluctuations (Schradin \& Pillay, 2004), resulting in changes in resource availability (Schradin, 2005), leading to changes in social organization 
(Schoepf \& Schradin, 2012; Schradin et al., 2010). Consequently, the environment that females will encounter as adults is unlikely to be the same as the one in which they were reared, leaving them unable to predict whether they will raise young with or without a mate (Schradin et al., 2010). Therefore, the ability of females to alter their behavior in response to changing environmental and social conditions could be highly beneficial for maximizing reproductive success and offspring survival. Under low population density, females favor solitary nesting to minimize the costs associated with reproductive competition (e.g., increased female-female aggression: Schubert et al., 2009; infanticide, Schradin et al., 2010). Under these conditions, organization of maternal hormones in inexperienced females could cause a heightened maternal response to offspring to maximize offspring survival. In contrast, when population density increases, females are often forced to nest in small groups because of the limited availability of nesting sites (Schradin \& Pillay, 2004; Schradin et al., 2010). Males typically show a nearly threefold increase in the time spent in the nest when pups are present (Schradin \& Pillay, 2003) and offspring development in this population is better when fathers are present (Schradin \& Pillay, 2005). Under these conditions, activational effects can trigger females to reduce their workload in response to males taking over much of the parental care duties.

Our study demonstrates the complex nature of maternal care expression in female striped mice. Females modify their behavior in response to their own internal state (possibly by hormonal regulation), their own experience of providing maternal care and their mate's motivation to provide paternal care (i.e., paternal care experience), indicating that the pathway for the behavioral expression of maternal care in striped mice is activated in response to prevailing social and environmental conditions. 


\section{Notes}

We are grateful to Megan Jones, Sneha Joshi and Melanie Schubert for providing technical assistance and to Davina Hill for critically reading the manuscript. Funding was provided by the National Research Foundation (grant number: 2069110; DOL Scarce Skills scholarship) and the University of the Witwatersrand.

\section{References}

Bredy, T. W., Lee, A. W., Meaney, M. J., \& Brown, R. E. (2004). Effect of neonatal handling and paternal care on offspring cognitive development in the monogamous California mouse (Peromyscus californicus). Hormones and Behavior, 46, 30-38.

Champagne, F., Diorio, J., Sharma, S., \& Meaney, M. J. (2001). Naturally occurring variations in maternal behavior in the rat are associated with differences in estrogeninducible central oxytocin receptors. Proceedings of the Natural Academy of Sciences USA, 98, 12736-12741.

Curley, J. P., Champagne, F. A., Bateson, P., \& Keverne, E. B. (2008). Transgenerational effects of impaired maternal care on behaviour of offspring and grandoffspring. Animal Behaviour, 75(4), 1551-1561.

Elekonich, M. M., \& Robinson, G. E. (2000). Organizational and activational effects of hormones on insect behavior. Journal of Insect Physiology, 46, 1509-1515.

Francis, D., Diorio, J., Liu, D., \& Meaney, M. J. (1999). Nongenomic transmission across generation of maternal behavior and stress responses in the rat. Science, 286(5442), $1155-1158$. 
Galea, L. A. M., Kavaliers, M., \& Ossenkopp, K-P. (1996). Sexually dimorphic spatial learning in meadow voles Microtus pennsylvanicus and deer mice Peromyscus maniculatus. Journal of Experimental Biology, 199, 195-200.

Goebelsmann, U., Roberts, J. M., \& Jaffe, R. B. (1972). Placental transfer of 3H-oestriol-3sulphate-16-glucosiduronate and 3H-oestriol-16-glucosiduronate-14C. Acta Endocrinologica, 70, 132-142.

Gubernick, D. J., \& Teferi, T. (2000). Adaptive significance of male parental care in a monogamous mammal. Proceedings of the Royal Society of London B: Biological Sciences, 267(1439), 147-150.

Kikusui, T., Isaka, Y., \& Mori, Y. (2005). Early weaning deprives mouse pups of maternal care and decreases their maternal behavior in adulthood. Behavioural Brain Research, 162, 200-206.

Jia, R., Tai, F., An, S., \& Zhang, X. (2011). Neonatal paternal deprivation or early deprivation reduces adult parental behaviour and central estrogen receptor $\alpha$ expression in mandarin voles (Microtus mandarinus). Behavioural Brain Research, 224, 279-289.

Libersat, F., \& Pflüger, H.-J. (2004). Monoamines and the orchestration of behavior. Bioscience, 54, 17-25.

Liu, D., Diorio, J., Tannenbaum, B., Caldji, C., Francis, D., Freedman, A., Sharma, S., Pearson, D., Plotsky, P. M., \& Meaney, M. J. (1997). Maternal care, hippocampal glucocorticoid receptors, and hypothalamic-pituitary-adrenal responses to stress. Science, 277(5332), 1659-1662.

McGuire, B. (1997). Influence of father and pregnancy on maternal care in red-backed voles. Journal of Mammalogy, 78(3), 839-849. 
Meaney, M. J. (2001). Maternal care, gene expression, and the transmission of individual differences in stress reactivity across generations. Annual Review of Neuroscience, 24, $1161-1192$.

Numan, M., \& Insel, T. R. (2003). The neurobiology of parental behaviour. New York: Springer.

Olazábal, D. E., \& Young, L. J. (2005). Variability in "spontaneous” maternal behaviour is associated with anxiety-like behaviour and affiliation in naïve juvenile and adult female prairie voles (Microtus ochrogaster). Developmental Psychobiology, 47, 166-178.

Oliveira, R. F. (2009). Social behavior in context: Hormonal modulation of behavioral plasticity and social competence. Integrative and Comparative Biology, 49, 423-440.

Outscharoff, W., Jr., Helmeke, C., \& Braun, K. (2006). Lack of paternal care affects synaptic development in the anterior cingulated cortex. Brain Research, 1116, 58-63.

Phoenix, C., Goy, R., Gerall, A., \& Young, W. (1959), Organizing action of prenatally administered testosterone propionate on the tissues mediating mating behavior in the female guinea pig. Endocrinology, 65(3), 369-382.

Prendergast, B. J., Zucker, I., \& Nelson, R. J. (2009). Seasonal rhythms of mammalian behavioral neuroendocrinology, in: D.W. Pfaff (Ed.), Hormones, Brain, and Behavior. Boston: Academic Press.

Rymer, T., \& Pillay, N. (2010). Female mate choice for paternal care behaviour in African striped mice Rhabdomys pumilio: the role of experience. Behaviour, 147, 1101-1119.

Rymer, T. L., \& Pillay, N. (2011). The influence of the early rearing environment on the development of paternal care in African striped mice. Ethology, 117, 284-293.

Soares, M. C., Bshary, R., Fusani, L., Goymann, W., Hau, M., Hirschenhauser, K., \& Oliveira, R. F. 2010: Hormonal mechanisms of cooperative behaviour. Philosophical Transactions of the Royal Society B: Biological Sciences, 365, 2737-2750. 
Schradin, C. (2005). When to live alone and when to live in groups: ecological determinants of sociality in the African striped mouse (Rhabdomys pumilio, Sparrman, 1784). Belgian Journal of Zoology, 135(supplement), 77-82.

Schradin, C., \& Pillay, N. (2003). Paternal care in the social and diurnal striped mouse (Rhabdomys pumilio): Laboratory and field evidence. Journal of Comparative Psychology, 117(3), 317-324.

Schradin, C., \& Pillay, N. (2004). The striped mouse (Rhabdomys pumilio) from the succulent karoo of South Africa: A territorial group living solitary forager with communal breeding and helpers at the nest. Journal of Comparative Psychology, 118(1), 37-47.

Schradin, C., \& Pillay, N. (2005). The influence of the father on offspring development in the striped mouse. Behavioral Ecology, 16, 450-455.

Schradin, C., Köenig, B., \& Pillay, N. (2010). Reproductive competition favours solitary living while ecological constraints impose group-living in African striped mice. Journal of Animal Ecology, 79, 515-521.

Schubert, M., Pillay, N., \& Schradin, C. (2009). Parental and alloparental care in a polygynous mammal. Journal of Mammalogy, 90, 724-731.

Schoepf, I., \& Schradin, C. (2012). Better off alone! Reproductive competition and ecological constraints determine sociality in the African striped mouse (Rhabdomys pumilio). Journal of Animal Ecology.

Skinner, J. D., \& Chimimba, C. T. (2005). The mammals of the southern African subregion. Cape Town: Cambridge University Press.

Song, Z., Tai, F., Yu, C., Wu, R., Zhang, X., Broders, H., He, F., \& Guo, R. (2010). Sexual or paternal experiences alter alloparental behaviour and the central expression of ER $\alpha$ and OT in male mandarin voles (Microtus mandarinus). Behavioural Brain Research, 214, 290-300. 
Stone, A. I. S., \& Bales, K. L. (2010). Intergenerational transmission of the behavioral consequences of early experience in prairie voles. Behavioural Processes, 84(3), 732-738.

Van den Bergh, B. R. H., Mulder, E. J. H., Mennes, M., \& Glover, V. (2005). Antenatal maternal anxiety and stress and the neurobehavioural development of the fetus and child: links and possible mechanisms. A review. Neuroscience and Biobehavioral Reviews, 29(2), 237-258.

Weladji, R. B., Gaillard, J. -M., Yoccoz, N. G., Holand, Ø., Mysterud, A., Loison, A., Nieminen, M., \& Stensth, N. C. (2006). Good reindeer mothers live longer and become better in raising offspring. Proceedings of the Royal Society of London B: Biological Sciences, 273, 1239-1244.

Wynne-Edwards, K. E. (1995). Biparental care in Djungarian but not Siberian dwarf hamsters (Phodopus). Animal Behaviour, 50(6), 1571-1585.

Young, W. C., Goy, R. W., \& Phoenix, C. H. (1964). Hormones and sexual behavior. Science, 143(3603), 212-218. 
Table 1. Predictors (treatment, time, treatment $x$ time, litter size) of maternal care displayed by female (mothers and daughters) striped mice.

\begin{tabular}{|c|c|c|}
\hline Predictors & Statistics & Post hoc comparisons \\
\hline \multicolumn{3}{|c|}{ Maternal care (Phase 1 vs. Phase 2) } \\
\hline \multirow[t]{3}{*}{ Treatment } & $F_{15,284.74}=7.71, p<0.001$ & Near: $\mathrm{M}+\mathrm{F}>\mathrm{DM}-\mathrm{F}=\mathrm{DM}+\mathrm{F}=\mathrm{DM} / \mathrm{F}>\mathrm{M}-\mathrm{F}>\mathrm{M} / \mathrm{F}$ \\
\hline & & Huddling: $\mathrm{M}-\mathrm{F}>\mathrm{M} / \mathrm{F}=\mathrm{DM}-\mathrm{F}=\mathrm{DM}+\mathrm{F}=\mathrm{DM} / \mathrm{F}>\mathrm{M}+\mathrm{F}$ \\
\hline & & Grooming: $\mathrm{M}-\mathrm{F}=\mathrm{M} / \mathrm{F}>\mathrm{DM}-\mathrm{F}=\mathrm{DM} / \mathrm{F}=\mathrm{DM}+\mathrm{F}=\mathrm{M}+\mathrm{F}$ \\
\hline \multirow[t]{6}{*}{ Time } & $F_{15,91}=2.56, p=0.003$ & Near: $($ PND11) $>($ PND9) $>$ (PND7, PND5, PND3, $($ PND1) \\
\hline & & Huddling: (PND1, PND3) $>($ PND7, PND5 $)>($ PND9 $)>($ PND11 $)$ \\
\hline & & Phase 1 Grooming: (PND9, PND5, PND7) $>($ PND3, PND11) $>$ \\
\hline & & (PND1) \\
\hline & & Phase 2 Grooming: (PND9, PND11, PND5, PND1) $>$ (PND3, \\
\hline & & PND7) \\
\hline Treatment $\mathrm{x}$ Time & $F_{75,440.09}=2.12, p<0.001$ & See text \\
\hline Litter size & $F_{3,103}=0.21, p=0.889$ & \\
\hline
\end{tabular}

Statistics $=$ GLM with a repeated measures design. Post hoc comparisons are provided for significant predictors (indicated in bold) for the main effects: homogeneous (nonsignificant) subsets are given in parentheses. $\mathrm{M}+\mathrm{F}=$ mothers and fathers raised young together, $\mathrm{M} / \mathrm{F}=$ mothers separated from fathers by a metal barrier and raised young alone, $\mathrm{M}-\mathrm{F}=$ mothers raised young alone, $\mathrm{DM}+\mathrm{F}=$ daughters from $\mathrm{M}+\mathrm{F}$ treatment, $\mathrm{DM} / \mathrm{F}=$ daughters from $\mathrm{M} / \mathrm{F}$ treatment, $\mathrm{DM}-\mathrm{F}$ = daughters from $\mathrm{M}-\mathrm{F}$ treatment; PND = postnatal day. 
Table 2. Mean ( \pm SE) growth rates for male and female offspring during the first 21 days after birth for treatments in two phases.

\section{Phase/ Male growth rate $\quad$ Female growth rate $\quad$ Statistics}

\section{Treatment}

\section{Phase 1}

$\begin{array}{llll}\mathrm{M}+\mathrm{F} & 0.082(0.002) & 0.082(0.002) & \text { Treatment: } F_{2,41}=1.82, p=0.175 \\ \mathrm{M} / \mathrm{F} & 0.086(0.006) & 0.088(0.005) & \text { Male vs. female: } F_{1,41}=0.44, p=0.512 \\ \mathrm{M}-\mathrm{F} & 0.086(0.003) & 0.086(0.003) & \text { Litter size: } F_{1,41}=1.63, p=0.209 \\ & & & \text { Sex ratio: } F_{1,41}=0.21, p=0.652\end{array}$

Phase 2

$\mathrm{DM}+\mathrm{F} \quad 0.079(0.001) \quad 0.078(0.001) \quad$ Treatment: $F_{2,27}=1.12, p=0.340$

$\mathrm{DM} / \mathrm{F} \quad 0.077(0.001) \quad 0.077(0.002) \quad$ Male vs. female: $F_{1,27}=1.11, p=0.301$

$\mathrm{DM}-\mathrm{F} \quad 0.083(0.004) \quad 0.085(0.003) \quad$ Litter size: $F_{1,27}=1.23, p=0.278$

Sex ratio: $F_{1,27}=0.02, p=0.840$

Statistics $=$ GLM with a repeated measures design. None of the predictors were significant $\mathrm{M}+\mathrm{F}=$ mothers and fathers raised young together, $\mathrm{M} / \mathrm{F}=$ mothers separated from fathers by a metal barrier and raised young alone, $\mathrm{M}-\mathrm{F}=$ mothers raised young alone, $\mathrm{DM}+\mathrm{F}=$ daughters from $\mathrm{M}+\mathrm{F}$ treatment, $\mathrm{DM} / \mathrm{F}=$ daughters from $\mathrm{M} / \mathrm{F}$ treatment, DM - F = daughters from $\mathrm{M}-\mathrm{F}$ treatment. 
Table 3. Predictors (treatment, time, treatment $x$ time, litter size) of maternal care displayed by female striped mice.

\begin{tabular}{lc}
\hline Predictors & \multicolumn{1}{l}{ Statistics } \\
\hline Maternal care (inexperienced vs. experienced vs. older male) \\
Treatment & $\boldsymbol{F}_{\mathbf{2 , 2 6}}=\mathbf{1 3 . 5 0}, \boldsymbol{p}<\mathbf{0 . 0 0 1}$ \\
Time & $F_{5,130}=0.45, p=0.812$ \\
Treatment x Time & $F_{10,130}=0.41, p=0.942$ \\
Litter size & $F_{1,26}=0.97, p=0.334$
\end{tabular}

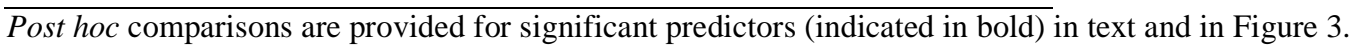

Inexperienced $=$ both males and females parentally inexperienced, Experienced $=$ both males and females parentally experienced, Older male $=$ females maternally inexperienced but males paternally experienced. Statistics $=$ GLM with a repeated measures design . 

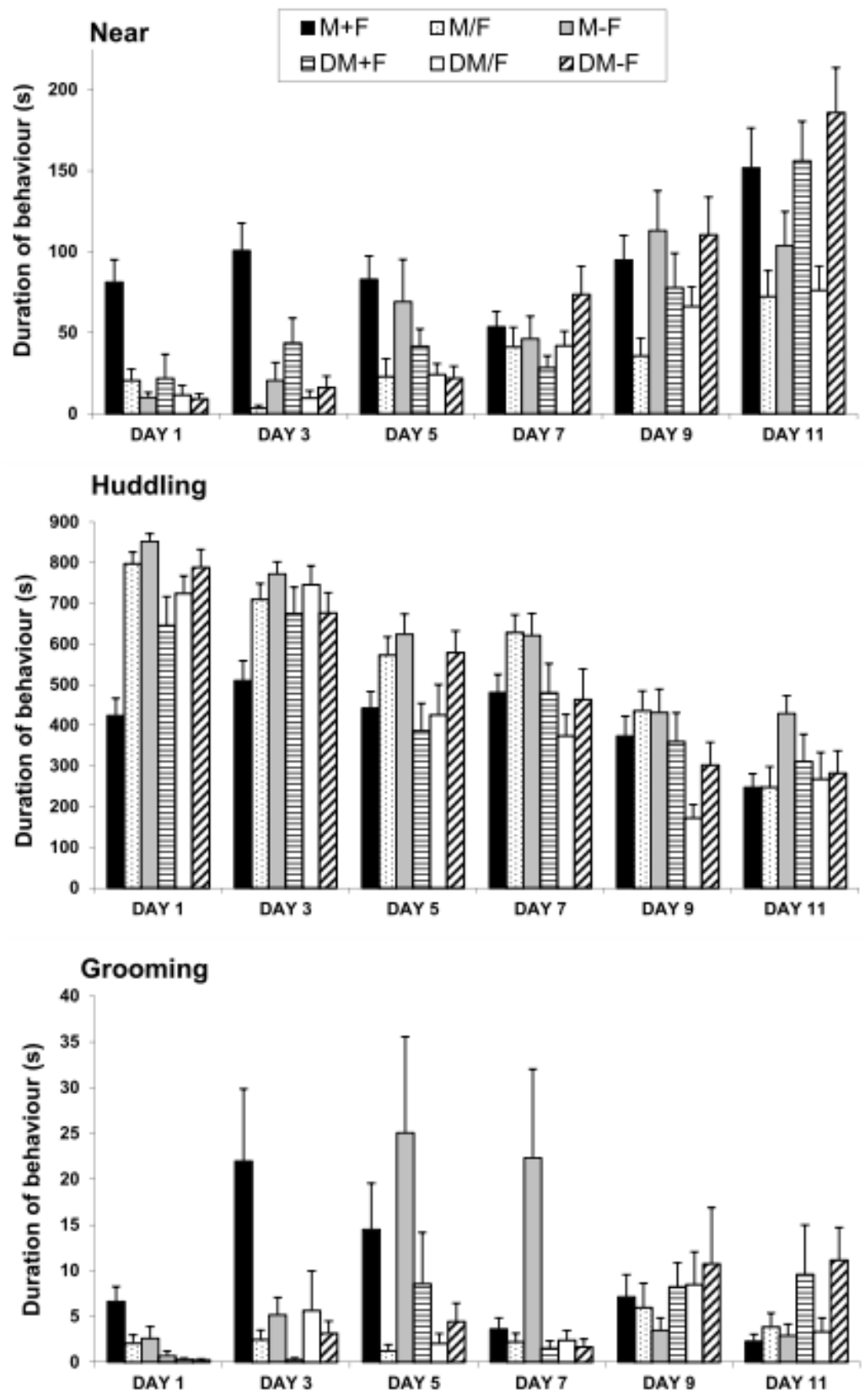

Figure 1 Mean \pm SE time (seconds) spent on three parental care behaviours (near, huddling, grooming) by female (mothers and adult daughters) striped mice for 6 taping days (Days 1 11). Significant differences (Fisher's HSD post hoc tests) are presented in Table $1 . \mathrm{M}+\mathrm{F}=$ mothers and fathers raised young together, $\mathrm{M} / \mathrm{F}=$ mothers raised young alone and separated from fathers by a metal barrier, $\mathrm{M}-\mathrm{F}=$ mothers raised young alone, $\mathrm{DM}+\mathrm{F}=$ daughters from $\mathrm{M}+\mathrm{F}$ treatment, $\mathrm{DM} / \mathrm{F}=$ daughters from $\mathrm{M} / \mathrm{F}$ treatment, $\mathrm{DM}-\mathrm{F}=$ daughters from $\mathrm{M}$ F treatment. 


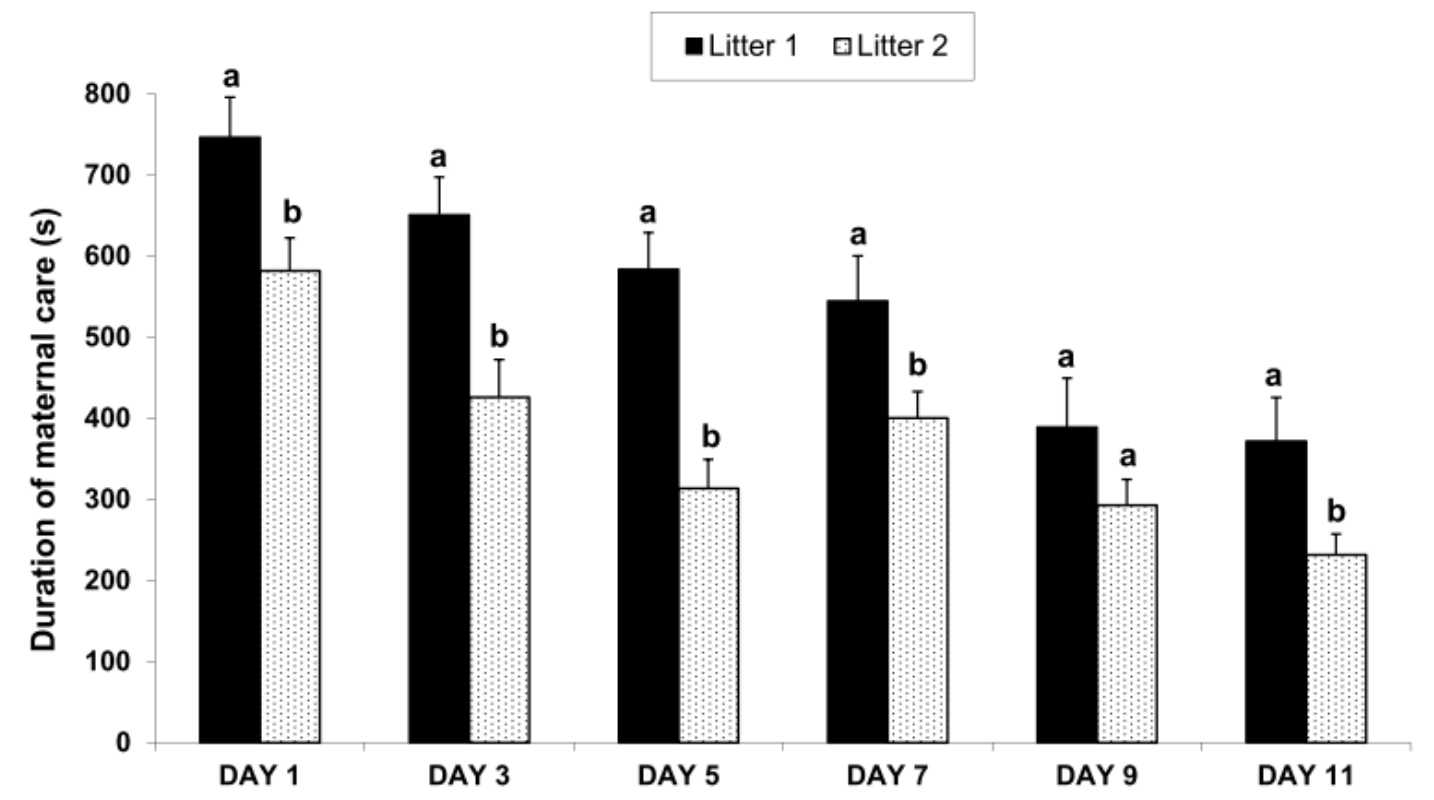

Figure 2 Mean \pm SE time (seconds) spent in maternal care by female striped mouse daughters for 6 taping days (Days 1 - 11) for two consecutive litters (Litter 1 and Litter 2). Significant differences (Fisher's HSD post hoc tests) are presented in text. 


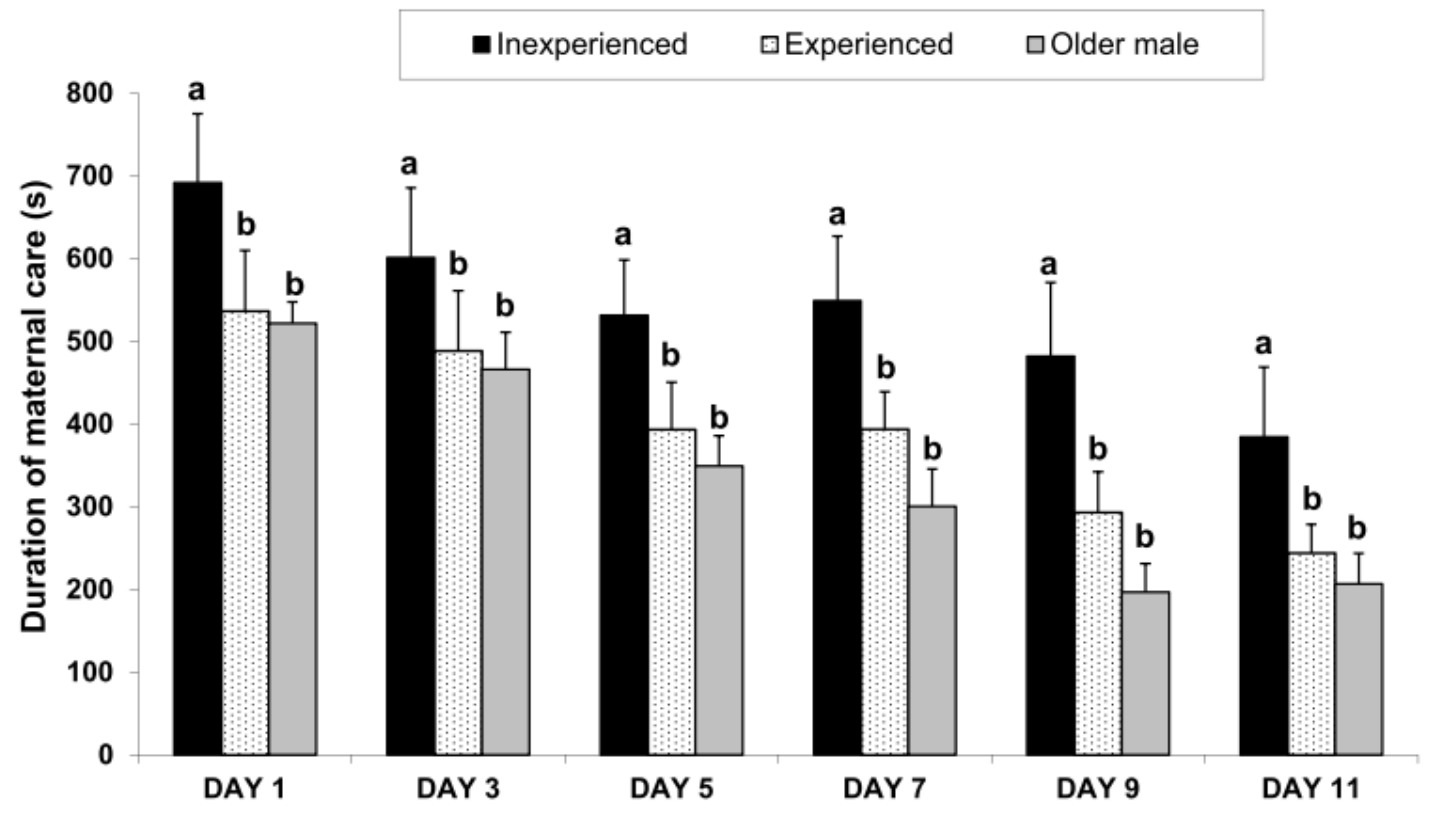

Figure 3 Mean \pm SE time (seconds) spent in maternal care by female striped mouse daughters for 6 taping days (Days $1-11$ ) when raising young with inexperienced, experienced or older males. Significant differences (Fisher's HSD post hoc tests) are presented in text. 